\title{
RENDIMIENTO ACADÉMICO DE SECUNDARIA Y SU RELACIÓN CON EL DE PRIMER AÑO DE EDUCACIÓN UNIVERSITARIA EN LA UN/JBG DE TACNA, EN EL AÑO 2006
}

\author{
Responsable \\ Miembro \\ Mgr. Dionicio Milton Chàvez Muñoz \\ Mgr. Julia Marina Mendoza Gómes
}

\begin{abstract}
RESUMEN
El objetivo de la presente investigación es determinar el grado de relación que existe entre el rendimiento académico de secundaria con el rendimiento académico alcanzado por el estudiante en el primer año de educación universitaria en la Universidad Nacional Jorge Basadre Grohmann de Tacna, en el año 2006. El método es empirico, basado en análisis documentario de rendimiento de ingreso y rendimiento alcanzado por los estudiantes según actas finales de evaluación. Las conclusiones a las que se arribó al finalizar esta investigación son: 1) El rendimiento académico de los estudiantes de secundaria al ingresar a la universidad se concentra en la categoria de regular a bueno, pues no hay desaprobados, con un $56,3 \%$ (regular) y un $43,1 \%$ (bueno). 2) El rendimiento académico de los estudiantes en el primer año de estudios universitarios se concentra en la categoria de regular con un $49,7 \%$ (regular) y un $17,9 \%$ (bueno). 3) El 32,4\% de estudiantes disminuyen su rendimiento académico en el primer año de universidad y pasan de la condición de aprobados al ingresar a la universidad a la condición de desaprobados.
\end{abstract}

\begin{abstract}
The objective of the present investigation is to determine the degree of relation that exists between the academic yield of secondary with the academic yield reached about the student in the first year of university education in the Universidad Nacional Jorge Basadre Grohmann of Tacna, in 2006; the method empirical is based on documentary analysis of yield of entrance and yield reached about the students according to final acts of evaluation: the conclusions at which this investigation was arrived when finalizing are: (1) The academic yield of the students of secondary when entering the university are concentrated in the category of regulating to good, because it does not have disapproved, with a $56.3 \%$ (to regulate) and a $43.1 \%$ (good). (2) The academic yield of the students in the first year of university studies is concentrated in the category of regulating with a $49.7 \%$ (to regulate) and $17.9 \%$ (good). (3) That $32.4 \%$ of students diminish their academic yield in the first year of approved university and happen of the condition of when entering the disapproved university to the condition of
\end{abstract}

\section{INTRODUCCION}

En las facultades de la Universidad Nacional Jorge Basadre Grohmann, es preocupante encontrar cada año un alto porcentaje de estudiantes desaprobados en las asignaturas de matemática.

Al mismo tiempo, los estudiantes que tienen la condición de aprobados se encuentran con notas muy bajas, como ser 11 ó 12, lo cual muestra que la aprobación está condicionada a la permanencia del estudiante en la universidad.

El estudiante lleva consigo el rendimiento alcanzado en la secundaria a la universidad en cocimientos previos, actitudes y habilidades para hacer frente a los estudios universitarios. Algunos conservan su rendimiento académico, pero otros bajan su nivel de rendimiento al cursar el primer año de universidad.

En este sentido se presenta un estudio sobre la relación que existe entre el rendimiento del estudiante al finaliza sus estudios secundarios con el alcanzado en el primer año de universidad en las carreras de ingenieria.

\section{PLANTEAMIENTO DEL PROBLEMA}

El rendimiento académico de los estudiantes del primer año de universidad se considera relativamente bajo en las diferentes facultades de la Universidad Nacional Jorge
Basade Grohmann. Este hecho tiene diferentes causas, entre las que podemos destacar: conocimientos previos, motivación, rendimiento académico, situación social, lugar de procedencia, colegio de procedencia, tiempo de dedicación al estudio, etc.

Sabido es que los estudiantes cada año disminuyen su rendimiento académico y en algunos casos para promocionar alumnos de un año a otro se tienen que bajar los niveles de exigencia, llevando consigo la correspondiente disminución de la calidad del profesional que se está formando.

Una de las causas de alta influencia es el nivel de rendimiento académico alcanzado por el estudiante en la secundaria, porque está relacionado con la preparación del joven y su pretensión de seguir una carrera universitaria.

En este sentido, se plante la cuestión siguiente:

¿Cómo se relacionan el rendimiento académico de secundaria con el rendimiento académico alcanzado por el estudiante en el primer año de educación universitaria en la UNJBG de Tacna, en el año 2006?

\section{1) Objetivos de Investigación}

\section{Objetivo General}

Determinar el grado de relación que existe entre el 
rendimiento académico de secundaria con el rendimiento académico alcanzado por el estudiante en el primer año de educación universitaria en la UNJBG de Tacna, en el año 2006.

\section{Objetivos Especificos}

Objetivo 01: Precisar el nivel de rendimiento académico alcanzado por el estudiante al finalizar la secundaria.

Objetivo 02: Precisar el nivel de rendimiento académico alcanzado por el estudiante en el primer año de universidad.

Objetivo 03: Precisar la relación que existe entre el rendimiento académico de los estudiantes ingresantes a la UNJBG y el alcanzado en el primer año de universidad.

\section{DISEÑO DE LAINVESTIGACIÓN}

La Población ha estado constituida por todos los ingresantes al primer año de la Universidad nacional Jorge Basadre Grohmann de Tacna.

La Muestra ha estado conformada por todos los ingresantes a las carreras de ingenieria.

Las Unidades de Análisis son los estudiantes del primer año de la UNJBG de Tacna, expresados en rendimiento al ingresar y al concluir su primer año.

\section{1) Hipótesis de Investigación}

\section{Hipótesis General.}

Existe relación entre el rendimiento académico de secundaria con el rendimiento académico alcanzado por el estudiante en el primer año de educación universitaria en la UNJBG de Tacna, en el año 2006

\section{Hipótesis Especificas}

Hipótesis 01: El rendimiento académico alcanzado por los estudiantes de secundaria es regular.

Hipótesis 02: El rendimiento académico logrado por los estudiantes en el primer año de universidad es regular.

Hipótesis 03: Existe relación entre el rendimiento académico de los estudiantes ingresantes a la UNJBG y el alcanzado en el primer año de universidad.

\section{2) Técnicas y Métodos de Trabajo.}

Las Técnicas. Para la recolección y tratamiento en el desarrollo de este trabajo se usaron:

- Trabajo de campo en las oficinas de Admisión, registros académicos de cada Facultad y OASA de la Universidad

- El análisis documental de informes académicos, actas finales de evaluación y registros de rendimiento académico de los estudiantes.
- La evaluación de la información y de los documentos.

El Método Estadistico. Para realizar el análisis, dar las interpretaciones cualitativas y cuantitativas de los resultados alcanzados. Se usó Exel, Estadística, para este propósito.

Los Instrumentos. Para este trabajo se usaron instrumentos que permitieron recabar y procesar la información. Se usaron:

- Cuaderno o libreta de apuntes (cuaderno de campo).

- Matrices de análisis con entrada doble y múltiple.

- Las escalas de evaluación educativa.

\section{ANTECEDENTES}

Se precisan las definiciones de rendimiento académico sugeridas por varios autores de rendimiento académico. Por un lado se define el rendimiento académico como el proceso técnico - pedagógico que juzga los logros de acuerdo con los objetivos de aprendizaje preestablecidos; por otro lado, se indica que el rendimiento académico es el nivel de progreso en las materias que son objeto de aprendizaje; también se precisa que es el resultado del aprovechamiento escolar en función de los objetivos escolares y hay autores que perciben el rendimiento académico como el éxito o fracaso en el estudio, expresado a través de notas o calificativos.

En el Perú la deserción y repitencia en la universidad muestran que el proceso de admisión no ha podido detectar quiénes realmente deben seguir estudios universitarios; un número significativo de alumnos no ha podido responder a las exigencias que le conduzcan a tener logros significativos en la universidad.

Es que los procesos de admisión no son los más idóneos para tan complicado proceso de seleccionar al estudiante que debe estudiar determinada carrera profesional.

Hay una tendencia a acumular a los estudiantes aprobados alrededor de la nota once (11) en mayor medida y doce (12) en segundo lugar. Para el caso de los estudiantes desaprobados están acumulados con nota siete (07), lo cual muestra una clara evidencia de apoyo para que este estudiante pase a tener una oportunidad de aprobación en aplazados.

Estos sucesos tienden a hacer distorsionar el real rendimiento académico de los estudiantes.

\section{ELANÁLISIS DE DATOS YDISCUSIÓN DE RESULTADOS}

Según los datos recolectados se elaboraron las informaciones que permitieron hacer el seguimiento del rendimiento académico de cada estudiante alcanzado en el examen de ingreso a la universidad, es decir al culminar sus estudios de secundaria, y el logrado por ellos mismos en el primer año de universidad en las Escuelas de Ingenieria de la UNJBG de Tacna. 
Como paso siguiente se hizo un análisis detallado de las informaciones elaboradas usando la estadistica y la teoria especializada sobre los temas abordados, para poder dar la información cuantitativa en cuadros estadisticos, en gráficas, en porcentajes, etc.

Las informaciones que se obtuvieron tuvieron, además, una interpretación cualitativa, para el adecuado entendimiento de los resultados, conclusiones y recomendaciones que se plantearon al final de la presente investigación.

Se debe precisar que el nivel logrado por el estudiante al finalizar la secundaria lo alcanza en el examen de ingreso a la universidad.

Finalmente, se detalla el logro de objetivos y la prueba de las hipótesis que son obtenidas a partir del análisis e interpretación.

\section{RESULTADOS DEL RENDIMIENTO DE LOS ESTUDIANTES}

\section{Discusión}

Presentamos aquí cuadros de resumen de los resultados de las escuelas estudiadas, el rendimiento de ingreso a la universidad en las diferentes escuelas académicoprofesionales y el rendimiento en el primer año de universidad en las diferentes escuelas académico . profesionales de ingeniería

Cuadro N $^{\circ} 01$ : De Resultados de las Escuelas Estudiadas.

\begin{tabular}{|c|c|c|c|}
\hline ESCUELA & Desaprobados & Aprobados & TOTAL \\
\hline ESGE & 2 & 5 & $\mathbf{7}$ \\
\hline ESIQ & 1 & 4 & $\mathbf{5}$ \\
\hline ESCO & 2 & 46 & $\mathbf{4 8}$ \\
\hline ESMC & 14 & 18 & $\mathbf{3 2}$ \\
\hline ESIC & 14 & 14 & $\mathbf{2 8}$ \\
\hline ESAU & 21 & 26 & $\mathbf{3 7}$ \\
\hline TOTAL & $\mathbf{5 4}$ & $\mathbf{1 1 3}$ & $\mathbf{1 6 7}$ \\
\hline & $\mathbf{3 2 , 3 4} \%$ & $\mathbf{6 7 , 6 6} \%$ & $\mathbf{1 0 0} \%$ \\
\hline
\end{tabular}

Interpretación. En este Cuadro No 01 observamos que el $32,34 \%$ de los estudiantes está en la condición de desaprobados y que el $67,66 \%$ está en la condición de aprobados

Cuadro $N^{\circ}$ 02: Rendimiento de los Estudiantes en Secundaria.

\begin{tabular}{|c|c|c|c|c|c|c|c|c|}
\hline \multicolumn{9}{|c|}{$\begin{array}{l}\text { RENDIMIENTO DE INGRESO A LA } \\
\text { UNIVERSIDAD EN LAS DIFERENTES } \\
\text { ESCUELAS ACADÉMICO - PROFESIONALES }\end{array}$} \\
\hline \multirow[t]{2}{*}{ CONDICIÓN } & \multirow{2}{*}{$\begin{array}{l}\text { E } \\
S \\
G \\
E\end{array}$} & \multirow{2}{*}{$\begin{array}{l}E \\
S \\
1 \\
Q\end{array}$} & \multirow{2}{*}{$\begin{array}{l}E \\
\text { S } \\
\text { C } \\
\text { O }\end{array}$} & \multirow{2}{*}{$\begin{array}{c}E \\
S \\
M \\
C\end{array}$} & \multirow{2}{*}{$\begin{array}{l}E \\
S \\
I \\
C\end{array}$} & \multirow{2}{*}{$\begin{array}{l}E \\
\mathbf{S} \\
\dot{A} \\
\mathbf{U}\end{array}$} & \multicolumn{2}{|c|}{ TOTAL } \\
\hline & & & & & & & $\mathbf{n}$ & $\%$ \\
\hline $\begin{array}{l}\text { MUY } \\
\text { MALO } \\
\text { OO-O6 }\end{array}$ & O & $\mathrm{O}$ & o & O & O & O & $\mathbf{0}$ & $\mathbf{0}$ \\
\hline $\begin{array}{c}\text { MALO } \\
07-10\end{array}$ & o & o & O & O & O & $\mathrm{O}$ & $\mathbf{0}$ & $\mathbf{0}$ \\
\hline $\begin{array}{c}\text { REGULAR } \\
11-13\end{array}$ & 6 & 3 & 16 & 24 & 6 & 39 & 94 & 56,3 \\
\hline $\begin{array}{l}\text { BUENO } \\
14-17\end{array}$ & 1 & 2 & 31 & 8 & 22 & 8 & 72 & 43,1 \\
\hline $\begin{array}{l}\text { MUY } \\
\text { BUENO } \\
18-20\end{array}$ & o & O & 1 & O & o & O & 1 & 0,6 \\
\hline TOTAL & 7 & 5 & 48 & 32 & 30 & 47 & 167 & 100 \\
\hline
\end{tabular}

Interpretación. En este Cuadro $\mathrm{N}^{0} 02$ observamos que no hay estudiantes en condición de desaprobados; que el 56,3\% de los estudiantes está en la condición de aprobados en el nivel de regular y el $43,1 \%$ aprobados en el nivel bueno.

Cuadro N03: Rendimiento de los Estudiantes en el Primer Año de Universidad.

\begin{tabular}{|c|c|c|c|c|c|c|c|c|}
\hline \multicolumn{9}{|c|}{$\begin{array}{l}\text { RENDIMIENTO EN PRIMER AÑO DE } \\
\text { UNIVERSIDAD EN LAS DIFERENTES } \\
\text { ESCUELAS ACADEMICO - PROFESIONALES }\end{array}$} \\
\hline \multirow{2}{*}{ CONDICIÓN } & \multirow{2}{*}{$\begin{array}{l}E \\
S \\
G \\
E\end{array}$} & \multirow{2}{*}{$\begin{array}{l}E \\
S \\
I \\
Q\end{array}$} & \multirow{2}{*}{$\begin{array}{l}E \\
\text { S } \\
\text { C } \\
O\end{array}$} & \multirow{2}{*}{$\begin{array}{c}E \\
S \\
M \\
C\end{array}$} & \multirow{2}{*}{$\begin{array}{c}\text { E } \\
\text { S } \\
\text { I } \\
\text { C }\end{array}$} & \multirow{2}{*}{$\begin{array}{l}\mathrm{E} \\
\mathrm{S} \\
\mathrm{A} \\
\mathrm{U}\end{array}$} & \multicolumn{2}{|c|}{ TOTAL } \\
\hline & & & & & & & $\mathbf{n}$ & $\%$ \\
\hline $\begin{array}{l}\text { MUY } \\
\text { MALO } \\
00-06\end{array}$ & 0 & 1 & 2 & 4 & 3 & 7 & 17 & 10,2 \\
\hline $\begin{array}{c}\text { MALO } \\
07-10\end{array}$ & 2 & $\mathrm{O}$ & $\mathrm{O}$ & 10 & 11 & 14 & 37 & 22,2 \\
\hline $\begin{array}{c}\text { REGULAR } \\
11-13\end{array}$ & 4 & 0 & 32 & 14 & 10 & 23 & 83 & 49,7 \\
\hline $\begin{array}{l}\text { BUENO } \\
14-17\end{array}$ & 1 & 4 & 14 & 4 & 4 & 3 & 30 & 17,9 \\
\hline $\begin{array}{l}\text { MUY } \\
\text { BUENO } \\
18-20\end{array}$ & 0 & 0 & 0 & 0 & 0 & $\mathrm{O}$ & $\mathbf{0}$ & $\mathbf{0}$ \\
\hline TOTAL & 7 & 5 & 48 & 32 & 28 & 47 & 167 & 100 \\
\hline
\end{tabular}

Interpretación. En este Cuadro $\mathrm{N}^{\circ} 03$ observamos que hay un $32,4 \%$ de estudiantes en condición de desaprobados y el $56,3 \%$ de los estudiantes están en la condición de aprobados. Hay un $22,2 \%$ en el nivel de malos, un $49,7 \%$ en el nivel de regular y un $17,9 \%$ en el nivel bueno.

Cuadro N04: Coeficiente de Correlación

\begin{tabular}{|c|c|}
\hline $\begin{array}{c}\text { RENDIMIENTO AL } \\
\text { FINALIZAR LA } \\
\text { SECUNDARIA }\end{array}$ & $\begin{array}{c}\text { RENDIMIENTO EN EL } \\
\text { PRIMER ANNO DE } \\
\text { UNIVERSIDAD }\end{array}$ \\
\hline 0 & 17 \\
\hline 0 & 37 \\
\hline 94 & 83 \\
\hline 72 & 30 \\
\hline 1 & 0 \\
\hline Coeficiente de Correlación de $\mathbf{0 , 7 6}$ \\
\hline
\end{tabular}

Interpretación. La observación de los cuadros 02 y 03 permite precisar que del grupo de alumnos que estaban en el nivel de buenos pasan al grupo de regulares en un $25,2 \%$; del grupo de regulares pasan al grupo de malos y muy malos un $32,4 \%$. Esto quiere decir que, en líneas generales, el rendimiento académico de los estudiantes ha decaído comparando al finalizar la secundaria con el primer año de universidad.

\section{Corroboración de Hipótesis}

En primer lugar, al hacer el análisis de la información, se llega a establecer que efectivamente el rendimiento académico de los estudiantes de secundaria, al ingresar a la universidad, es regular, el rendimiento académico es valorado mayormente con calificaciones de 11 ó 12, lo cual permite confirmar la hipótesis especifica 1.

En segundo lugar, se llega a establecer que efectivamente el rendimiento académico de los estudiantes en el primer año de universidad se agrupa en el nivel de regular con inclinación hacia el grupo de malos; el grupo de regular disminuye de $43,1 \%$ al $17,9 \%$, lo cual permite corroborar la hipótesis especifica 2. 
En tercer lugar, se llega a establecer que efectivamente el rendimiento académico de los estudiantes de secundaria, al ingresar a la universidad, está relacionado con el rendimiento académico del primer año de universidad, con un coeficiente de correlación de 0,76, lo cual permite corroborar la hipótesis específica 3.

\section{CONCLUSIONES}

1. El rendimiento académico de los estudiantes de secundaria al ingresar a la universidad se concentra en la categoria de regular a bueno, pues no hay desaprobados, con un $56,3 \%$ (regular) y un $43,1 \%$ (bueno).

2. El rendimiento académico de los estudiantes en el primer año de estudios universitarios se concentra en la categoría de regular con un $49,7 \%$ (regular) y $17,9 \%$ (bueno).

3. El $32,4 \%$ de los estudiantes disminuyen su rendimiento académico en el primer año de universidad y pasa de la condición de aprobados al ingresar a la universidad a la condición de desaprobados.

4. El $67,6 \%$ de los estudiantes mantiene su rendimiento académico en el primer año de estudios en la universidad respecto al rendimiento académico que obtuvieron al ingresar.

5. Entre ambos rendimientos académicos, al ingresar a la universidad y en el primer año de universidad, existe una relación significativa, con una correlación de 0,76.

\section{BIBLIOGRAFIA}

Carballo Ramos, Elme y Barroto Pérez, Maria (2005): La Gestión Educativa en la Formación de Estudiantes.

Colectivo de Autores. Cepes. (1998): Los Métodos Participativos ¿Una nueva Concepción de la Enseñanza? Colectivo de Autores. Cuba.

Delgado, Kenneth y Cárdenas, Gerardo (2004) Aprendizaje Eficaz y Recuperación de Saberes . Editorial San Marcos. Lima.

Díaz-Barriga, F. y Hernández, G. (2002). Estrategias docentes para el aprendizaje significativo, una interpretación constructivista. Editorial Mc-GrawHill. México.

Gonzales Pérez, Miriam (2000): Evaluación del aprendizaje en la Enseñanza Universitaria. Revista Pedagógica Universitaria. Universidad de la Habana, Cuba

Peñaloza Ramella, Walter (2003): Los Propósitos de la Educación. Fondo Editorial del Pedagógico San Marcos. Lima.

Pozo, J. I. (1990). Estrategias de Aprendizaje. En Palacios, J., Marchesi, A. y Coll, C. (Comp.) Desarrollo Psicológico y Educación. Tomo I: Psicología Evolutiva. Madrid: Alianza Editorial, S.A., Capítulo 12, pp. 199-221

Pujol Balcells, Jaime (1981): Los métodos en la enseñanza universitaria, 2da edición. Ediciones Universidad de Navarra. Pamplona. 